\title{
Management international
}

International Management

Gestiòn Internacional

\section{Management de l'innovation et Globalisation - Enjeux et} pratiques contemporains sous la directions de : Ben Mahmoud-Jouini, S., Charue-Duboc F., Midler C., Dunod 2015

\section{David Massé}

Volume 19, numéro 4, été 2015

URI : https://id.erudit.org/iderudit/1043086ar

DOI : https://doi.org/10.7202/1043086ar

Aller au sommaire du numéro

Éditeur(s)

HEC Montréal

Université Paris Dauphine

ISSN

1206-1697 (imprimé)

1918-9222 (numérique)

Découvrir la revue

Citer ce compte rendu

Massé, D. (2015). Compte rendu de [Management de l'innovation et Globalisation - Enjeux et pratiques contemporains sous la directions de : Ben Mahmoud-Jouini, S., Charue-Duboc F., Midler C., Dunod 2015]. Management international / International Management / Gestiòn Internacional, 19(4), 199-202. https://doi.org/10.7202/1043086ar d'utilisation que vous pouvez consulter en ligne. 


\title{
Management de l'innovation et Globalisation - Enjeux et pratiques contemporains sous la directions de : Ben Mahmoud-Jouini, S., Charue-Duboc F., Midler C., Dunod 2015.
}

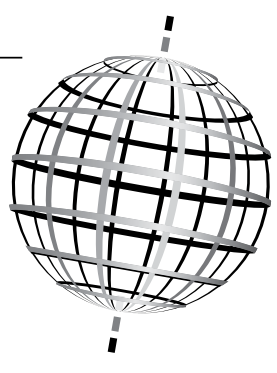

\author{
DAVID MASSÉ
}

$\mathrm{L}$ 'ouvrage «Management de l'innovation et globalisation » soulève un grand nombre d'enjeux d'actualités propres aux firmes multinationales dans un contexte d'innovation. Comme l'exprime Stéphane Richard dans la préface du livre, ces enjeux sont souvent abordés dans les médias de manière «incantatoire» ou «schématique». D'un côté, le thème est considéré comme le fer de lance des grands groupes occidentaux pour résister à la compétition des pays émergents et d'un autre, il fait planer le risque de délocalisation d'une main-d'œuvre qualifiée. Pour ne pas tomber dans ces écueils, le parti pris de ce livre est de partir du terrain et des questionnements empiriques vécus dans des entreprises internationales qui se résument en deux principaux axes de réflexions. D'une part, on assiste au développement de marchés émergents à forte croissance qui suppose d'adapter les offres de l'entreprise aux spécificités de ces marchés. D'autre part, l'internationalisation des entreprises favorise différentes formes de répartition des efforts de R\&D à travers le monde et pose aussi la question des incitatifs et des structures à mettre en place, afin de tirer parti des ressources réparties globalement. D'un point de vue théorique, la thématique de l'ouvrage se retrouve à l'articulation de deux champs (le management de l'innovation et le management international), mobilisés dans les analyses et pour lesquelles l'ouvrage a l'ambition de contribuer.

Le livre est construit sur la base de douze études de cas d'entreprises françaises œuvrant à l'international. Léchantillon est très riche, tant par la diversité des secteurs étudiés, que de la taille des entreprises. Au fil de la lecture, on passe de grandes entreprises dans l'industrie automobile (Renaud, Valéo), de la santé (Sanofi, Essilor), du gaz pour l'industrie (Air liquide), du divertissement (Ubisoft) à une strat-up dans la pâtisserie de Luxe (Hugo et Victor). L'effort de caractérisation des spécificités de chaque entreprise participe à la généralisation et à l'opérationnalisation des résultats mis en avant à un contexte plus large. La nationalité française de l'échantillon participe aussi à l'originalité de l'analyse et singularise cet ouvrage par rapport aux recherches existantes sur le sujet (Doz et Kosonen, 2008; Govindarajan et Trimble, 2012; Nadjou et al. 2012). Les réflexions proposées donnent en un seul livre un aperçu saisissant de la diversité des enjeux posés par cette thématique.
Il est aussi important de souligner que l'ouvrage est le fruit d'un travail collectif entre étudiants, praticiens et enseignants chercheurs dans le cadre du Master Projet innovation Conception offert par l'École polytechnique, HEC Paris et TelecomParisTech. La pédagogie de cette formation consiste à immerger pendant plus d'un an des étudiants en entreprises sur des projets innovants. Les cours du master permettent aux étudiants d'affiner leurs analyses de terrain à travers des allers - retours constants entre pratique et théorie. Il s'agit d'un dispositif de recherche et d'enseignement inédit qui permet un accès à des terrains et des données privilégiées.

Le livre se structure en trois grands thèmes : les processus de déploiement des innovations dans une firme multinationale, les enjeux et modes de l'adaptation locale, le développement et l'animation des forces de $\mathrm{R}$ et $\mathrm{D}$ mondialement réparties. Chaque thème est traité grâce à l'analyse de trois à cinq études de cas d'entreprise qui permettent d'éclairer un angle particulier de chaque thématique.

Le premier thème traître des processus de déploiement des innovations dans une firme multinationale. La notion de déploiement est définie comme «l'enchaînement des mises sur le marché d'une innovation par des filiales situées dans des contextes locaux différenciés, et nécessitant à chaque fois une adaptation voire une reconception de l'innovation d'origine» (p.3). Le parti pris de ce premier thème est de s'intéresser à des cas d'industries qui ne commercialisent pas des produits globaux mais qui doivent à la fois adapter leurs produits à des environnements locaux tout en tirant parti d'effets d'échelle. La question de la globalisation percute directement la question du management de l'innovation en faisant émerger la tension entre adaptations à des marchés locaux et intégration globale au sein de la firme.

À travers le cas de l'entreprise Air liquide, le premier chapitre analyse les facteurs critiques d'un déploiement massif et rapide. A partir de l'analyse de cinq déploiements d'innovation d'une des divisions du groupe, quatre facteurs de succès sont mis en avant : (1) l'existence d'un acteur qui pilote le déploiement de l'innovation, (2) l'existence de relation de co-développement avec des partenaires locaux, (3) la mise en place d'un réseau d'expert locaux et (4) le 
profil des filiales dans la première filiale dans laquelle l'innovation est commercialisée. Au-delà des spécificités de l'entreprise étudiée, cette analyse éclaire la problématique de l'internationalisation de l'innovation dans un contexte Business to Business avec une forte autonomie des filiales.

Le deuxième chapitre analyse le cas d'Orange à travers sa stratégie de développement dans la région Afrique et Moyen-Orient. L'étude se base sur l'analyse de la segmentation du portefeuille d'innovations de la firme et met en lumière une distinction entre 3 types d'innovation : les innovations globales (faible adaptation locale), les innovations locales (entièrement développées localement), les innovations régionales (naissance dans un pays et diffusion à une région d'un autre pays dont les besoins sont analogues). Cette étude montre qu'il y a coexistence de ces types d'innovations avec une gestion en portefeuille, à chaque type d'innovation correspond un mode de déploiement particulier ou les acteurs locaux et centraux jouent des rôles différents.

En se basant sur l'étude de la branche grande industrie de l'entreprise Air Liquide, le troisième chapitre contribue à catégoriser les types de filiales et de leurs rôles dans l'innovation. L'analyse fait ressortir quatre types de filiales : les grandes filiales historiques du groupe (par exemple, la France), les «implémenteurs» (par exemple, les pays de l'Europe de l'Est), les accélérateurs qui sont des pays de petite taille de marché, mais dont les clients sont extrêmement exigeants (la Suisse et l'Australie, par exemple), enfin les forts potentiels qui sont les grands pays émergents. Le modèle traditionnel de l'innovation suppose de concevoir l'innovation puis de l'introduire commercialement, les «implémenteurs» la déploient à l'identique en faisant profiter la firme d'économie d'échelle. Lidée que suggère cette typologie est que l'on peut imaginer d'autres types de trajectoires dans le déploiement de l'innovation, par exemple, cibler des besoins dans les pays émergents et produire dans un second temps dans les pays historiques, ou alors considérer les accélérateurs comme des incubateurs des pays à forts potentiels.

Le chapitre 4 se base sur l'analyse du cas Sanofi et illustre la manière dont une entreprise peut déployer à l'échelle internationale des services associés à une innovation dépendante de caractéristiques spécifiques des populations locales (contraintes administratives et réglementaires, par exemple). Des recherches dans le secteur de la santé (Denis et al. 2002) proposent de distinguer le noyau dur de la solution et sa périphérie malléable. Par exemple, si l'on considère l'application de jeu vidéo d'e-santé développé par Sanofi, il y au cœur de la solution, le jeu vidéo (la technologie à développer) et dans la périphérie, l'interface avec la nécessité d'envoyer des messages dans la langue du pays. Les résultats de ces travaux montrent qu'il faut distinguer une frontière claire entre ce qui est au coeur et ce qui est dans la périphérie et que plus la périphérie est large, plus on a des potentialités d'adaptation et des opportunités de déploiement. L'étude effectuée ne vérifie pas ce dernier point, car plus on a une périphérie large plus le coût d'adaptation est élevé. Cependant, le cas Sanofi montre qu'il est possible de distinguer deux types de solutions. D'une part des solutions qui sont relativement spécifiques à un certain type de pathologies et de patient avec une périphérie malléable et relativement limitée. D'autre part, des solutions noyau ou plateforme, c'est-à-dire une solution générique.

Enfin, le chapitre 5 s'appuie sur le cas Essilor dont l'expertise est distribuée entre plusieurs sites de production, de R\&D et de différentes filiales. La problématique abordée est la gestion des connaissances dans une organisation décentralisée. En effet, les apprentissages des bonnes pratiques et des connaissances produites localement restent le plus souvent dans la filiale et ne se diffusent pas dans l'organisation. Ainsi, le chapitre analyse la mise en place et l'animation d'une plateforme de partage en ligne pour favoriser les échanges réguliers malgré les distances géographiques. Trois facteurs clés de succès sont soulignés dans le développent de cette communauté d'expert : (1) le choix d'un outil approprié qui a permis une réponse aux demandes des premiers utilisateurs, (2) l'interdépendance entre le développement de la communauté et la mise au point de l'outil et (2) l'intéressement des différentes parties prenantes à l'utilisation de l'outil.

La deuxième partie de l'ouvrage approfondit les enjeux et les modes de l'adaptation locale des firmes multinationales. Plus courte que la première, cette partie mobilise trois études de cas.

Premièrement, à travers l'analyse du cas Valeo, le chapitre 6 examine l'exemple d'une entreprise qui tente d'élargir son offre à destination du Brésil à partir d'une R\&D localisée en Europe. Le groupe Valeo a mis en place une entité d'exploration, indépendante de la R\&D pour prendre en charge l'exploration de ces innovations de rupture à destination de ce marché local. Le problème est que les filiales ayant les connaissances sur les spécificités locales sont en relation avec les familles de produits, la $R \& D$, la vente... et non avec la cellule d'exploration qui réfléchit à des innovations en rupture. Ce qui a pour conséquence que lorsque des signaux sont émis, ils ne le sont pas au bon département et ils n'arrivent jamais au bon interlocuteur. Ainsi, la solution à ce problème a été de faire le lien entre les filiales locales pour les impliquer dans le processus d'exploration. Le problème est aussi que les entités d'explorations n'ont pas pour mission de réfléchir à des nouveaux produits pour les marchés émergents. On multiplie ainsi les obstacles, car il faut travailler avec un client global (Renault, par exemple) et sa filiale locale.

Le chapitre 7 porte sur le cas du véhicule électrique et de la stratégie ambitieuse développée par Renault. Le véhicule électrique a la propriété d'avoir un fort ancrage territorial notamment en raison des systèmes de recharge de ce produit. Il existe différents types de politiques publiques 
pour encourager (ou non) le déploiement d'une innovation. $\mathrm{Au}$ niveau des villes, il existe des leviers d'actions très différents de ceux au niveau de l'état qui sont par exemple des voies pour la circulation et des parkings réservés aux véhicules électriques. Ainsi, ce chapitre montre comment construire une méthodologie d'analyse d'un contexte local de l'innovation pour aider son adaptation et son déploiement.

La deuxième partie se termine par un chapitre traitant d'une entreprise singulière dans cet ouvrage, aussi bien à cause de sa taille que son secteur. Il traite d'une startup qui œuvre dans le domaine de la pâtisserie de luxe. Hugo \& Victor est une de ces jeunes entreprises qui depuis sa naissance expérimente de nouveaux marchés à l'international. De par sa petite taille, elle dispose d'une certaine agilité qui lui permet de proposer une offre «cœur» qui fonde l'identité de l'entreprise et des adaptations locales variées (logistiques, production, business model ...).

La troisième partie du livre aborde le développent et l'animation des forces de R\&D qui sont mondialement réparties.

Elle commence avec un chapitre qui aborde le cas Ubisoft, un développeur et éditeur de jeu vidéo dont les 29 studios sont répartis dans 19 pays. L'exemple traité dans le chapitre analyse une initiative de boîte à idées menée dans les studios français avec de possibles implications dans l'internationalisation de cette expérience.

Le chapitre 10 s'appuie sur le cas Orange à travers l'analyse de ses centres de recherche répartis à travers le monde. L'étude analyse ces centres à travers trois critères (la capacité productive, les liens avec le reste de l'entreprise et avec l'écosystème local) et propose une typologie de centres qui permet de souligner les rôles différenciés de ces centres dans les processus d'innovation de la firme. Il y a des entités qui sont très centrées sur des technologies spécialisées (les centres experts), il y a des unités qui ont un capital de ressource pour innover de manière variée (les powerhouse) et enfin des unités qui sont liées généralement à des gros marchés à bas coût (les lowcost).

Le chapitre 11 analyse le cas de Valeo en Chine et montre l'enjeu en matière de ressource humaine. L'entreprise a très tôt parié sur la chine qui se caractérise par un phénomène d'hypercroissance du marché. Il a donc fallu créer des unités d'appui pour adapter localement les produits de l'entreprise, mais aussi pour résoudre tous les problèmes de qualité et de fiabilité des produits. Historiquement, Valeo s'est déployé à l'international par une stratégie de croissance externe (rachat d'usines). La différence avec la chine est un changement d'échelle, de vitesse et de contexte. À l'origine le modèle d'internationalisation reposait sur une formalisation et sur une standardisation des processus de développement. Cette intégration a permis de garder une identité tout en maintenant la forte croissance de l'entreprise. Mais face au contexte chinois, ce modèle ne marche plus. Plusieurs enjeux ont ainsi émergé : (1) être capable de recruter massivement et rapidement en chine, (2) attirer des étudiants et les ingénieurs sur un marché ou l'entreprise est méconnue, (3) former les talents dans un contexte de barrières culturelles importantes, (4) et pour finir, retenir les talents dans les usines du groupe. Face à ces différents challenges, il y a nécessité de construire un projet RH nouveau et ambitieux afin d'appuyer un rythme de croissance diffèrent des autres pays.

Le dernier chapitre analyse une expérience de collaboration entre Renault et sa filiale Samsung motors. En effet, impliquer cette filiale coréenne dans le développement d'innovations liées à l'interface et aux produits de connexion pouvait être intéressant du fait de son écosystème. L'étude montre cependant plusieurs problèmes de coordination entre le technocentre de Renault et les centres de recherche de Samsung motors en Corée qui trouvent leurs origines notamment dans des difficultés de l'utilisation de l'anglais dans les deux pays induisant des problèmes de compréhension sur la sémantique des mots et dans un décalage au niveau des processus décisionnels.

La lecture des différents chapitres est très stimulante. Elle nous pousse à réfléchir de manière approfondie sur la base de nombreuses histoires d'internationalisation de processus de conception. Pour autant elle invite aussi à émettre quelques remarques critiques et laisse ouvertes quelques questions.

Tout d'abord, une première remarque a trait à la structure de l'ouvrage qui regroupe trois thématiques (déploiement, adaptation locale et développement/animation des forces de R\&D) qui donnent une apparente structure à l'ouvrage, mais qui ne se retrouve pas à la lecture des différents chapitres. La richesse empirique des exemples étudiés et la manière de les analyser apportent le plus souvent un éclairage beaucoup plus large que la thématique dans laquelle le chapitre s'insère. Il est en particulier difficile de comprendre l'attribution de certains chapitres entre les deux premiers thèmes tant les thématiques de déploiement et d'adaptation locales sont imbriquées dans l'analyse des différents cas. Enfin, le choix de présenter le cas Ubisoft à travers une expérience de boîte à idées réalisée au sein des studios français (et non étrangers) apparaît très étonnant (surtout si l'on considère la forte internationalisation de l'entreprise), et complètement déconnecté de la thématique traitée par le livre.

La première question concerne l'utilisation du mécanisme de la concurrence intra-firme qui peut dans certains cas justifier économiquement l'internationalisation de l'innovation. La question de la concurrence entre les différentes filiales et projet de R\&D d'un groupe apparaît peu dans les analyses présentées. En filigrane, elle est évoquée dans certains cas, mais elle semble perçue comme un frein au partage de connaissances et à la bonne coordination des filiales du groupe. Or, elle peut aussi être un des moteurs économiques qui pousse à la dissémination des ressources 
d'innovation à travers le globe. Elle repose sur le vieux mécanisme qui consiste à mettre en concurrence les équipes et les projets, c'est-à-dire provoquer une saine émulation de type : «qui sera capable d'innover, plus vite, et mieux que les autres? ». Il se pose ainsi la question de savoir comment organiser la concurrence entre les différentes équipes à travers la planète? L'organisation de la concurrence intrafirme fait cependant émerger une injonction paradoxale entre les différentes filiales du groupe («coopétition» intrafirme) et pose la question de savoir comment réinjecter des briques de collaboration et de partage dans un contexte de concurrence.

La deuxième question revoie à la dimension financière et à la gouvernance à mettre en place dans l'internationalisation de l'innovation d'une firme. De nombreux exemples montrent l'importance des systèmes d'incitation à l'innovation dans les firmes multinationales. Or, il semble intéressant d'explorer le lien entre les systèmes de financement de l'innovation et la réussite des innovations, c'est à dire à travers son adaptation, sa vitesse de déploiement, sa performance de marché, etc. Le lien soulève les questions suivantes : dans quel contexte le siège doit-il financer l'innovation? Dans quel contexte faut-il déléguer le financement de l'innovation en locale? Dans le cas d'une trajectoire d'innovation locale qui devient globale de par son utilisation dans l'ensemble de l'entreprise, quels sont les mécanismes de récompense et d'incitation à mettre en place dans les filiales? Primes individuelles? Augmentation de budgets? Visibilités des filiales dans l'entreprise? Autant de mécanismes d'incitations qui mériteraient d'être explorés.

La troisième question renvoie au caractère géopolitique de l'internationalisation de l'innovation. Au-delà de l'argument de la captation d'un nouveau marché, il y a des zones géographiques incontournables pour des grands groupes de certaines industries. Par exemple, au cours de sa croissance, Ubisoft a très rapidement établi une filiale de l'entreprise au Japon pour distribuer ses jeux dans le pays, mais surtout se rapprocher de gros acteurs de l'industrie comme Sony ou Nintendo. Au contraire, l'internationalisation de l'entreprise à Montréal a été motivée par des avantages fiscaux dans une ville ou l'industrie du jeu vidéo était à l'origine inexistante. La capacité d'Ubisoft à tirer parti des spécificités locales fait aujourd'hui de Montréal, une ville importante dans l'industrie de jeu vidéo. La notion de cluster ou de ville créative apparaît ainsi intéressante pour appréhender ce phénomène et amène les questionnements suivants : faut-il suivre le mouvement ou se singulariser géographiquement? Comment une firme peut-elle capitaliser sur l'écosystème local d'une filiale pour innover?

Les critiques formulées ne remettent pas en cause la qualité des réflexions proposées qui contribuent de manière importante aux deux champs théoriques mobilisés. Au management de l'innovation, elles apportent le fait de s'intéresser à l'aval des processus de développement notamment grâce à la mise en avant de la notion de déploiement. Elles contribuent aussi au management international en raffinant les multiples configurations de relations qu'il peut y avoir entre le centre et la périphérie. Il s'agit donc d'un livre qu'il convient de distiller, tant il comprend de matières prometteuses pour la recherche en innovation, en management international, mais aussi pour les praticiens qui y trouveront de nombreuses inspirations.

\section{Bibliographie}

Denis, J.-L., Hébert, Y., Langley, A., Lozeau, D. et Trottier, L.-H., 2002. "Explaining Diffusion Patterns for Complex Health Care Innovations", Health Care Management Review. Vol. 27, n³, pp. 60-73.

Doz Y., Kosonen M., 2008, Fast Strategy : How Strategic Agility will help you stay ahead of the game, Wharton School Press.

Govindarajan V., Trimble C., 2012, Reverse Innovation : Create far from home, win everywhere, Harvard Business Press.

Radjou N., Prabhu J., Ahuja S., 2012, Jugaad Innovation : Think Frugal, Be Flexible, Generate Breakthrough Growth, John Wiley $\&$ Sons. 